\section{The biological reducing agent Oxyrase improves the resuscitation of dormant Mycobacterium smegmatis and Mycobacterium avium subsp. paratuberculosis}

\author{
Kelly E. Rock, Lauren C. Kinkead, \\ Timothy E. Secott \\ Department of Biological Sciences, \\ Minnesota State University, Mankato, \\ Minnesota, USA
}

\begin{abstract}
Mycobacterium avium subsp. paratuberculosis (MAP) is the etiologic agent of Johne's disease, a chronic intestinal disease of cattle and other ruminants. Diagnostic culture for MAP is typically unrewarding until latter stages of the disease. This may be a consequence of oxidative damage to dormant organisms, which results from culture. The purpose of this investigation was to determine the effect of the commercial reducing agent 0 xyrase ${ }^{\circledR}$ for Broth (OB) on the recovery of dormant Mycobacterium smegmatis (MS) and MAP. Dormant organisms were inoculated into medium only or that supplemented with media containing serial dilutions of OB. Growth was monitored by optical density for up to 21 days. Treatment of MAP and MS with OB led to significant increases in recovery and growth yield. However, the concentration of $\mathrm{OB}$ necessary to promote recovery was dependent on the number of viable organisms present in seed cultures. Mitigating oxidative damage by using OB can facilitate the recovery of dormant mycobacteria. Whether this is a direct or indirect effect has yet to be established.
\end{abstract}

\section{Introduction}

Johne's disease, which is caused by the acid-fast bacillus Mycobacterium avium subsp. paratuberculosis (MAP), is a chronic intestinal disease that primarily affects domestic and wild ruminants worldwide. Johne's disease is particularly threatening to the economic viability of dairy farming in the US, wherein nearly $22 \%$ of the dairy herds are infected with MAP. ${ }^{1}$ Cattle are usually infected shortly after birth and do not display signs of the disease until two or more years after the initial infection. $^{2}$ Because infection with MAP, which is shed in feces, is not reliably detected until hosts have entered the clinical stage of the dis- ease,${ }^{3}$ one infected animal can infect many others in the herd before the first clinical case of Johne's disease is observed. It has been estimated that by the time the first animal develops clinical signs of Johne's disease, there may be as many as 20 other MAP-infected animals in the herd, ${ }^{2}$ although this estimate is controversial. ${ }^{4}$ The disease can be spread from herd to herd through the purchase of animals with undetectable infections. ${ }^{5}$

Following its establishment in the host, pathogenic mycobacteria are sequestered in granulomas in immunocompetent hosts. ${ }^{6}$ Within these granulomas, it is believed that MAP, an aerobic organism, enters a dormant state as a result of nutrient and/or oxygen limitation. ${ }^{7-9}$ When these dormant organisms are cultured directly in nutrient-rich media, they typically fail to grow. The exact reason for this is unclear. Work with other organisms has shown that dormant organisms are particularly susceptible to oxidative damage, and the sudden increase in aerobic metabolic rate may result in the formation of levels of oxygen free radicals that the organism at that instant is not capable of detoxifying. ${ }^{10,11}$ Indeed, the inclusion of scavengers of reactive oxygen species, namely pyruvate and catalase, in culture media improved the recovery of viable but nonculturable (VNC) Vibrio vulnificus. ${ }^{10}$

Because progression into the clinical phase of Johne's disease results from MAP having resumed active growth within the host, the probability that MAP will be recovered from cattle exhibiting clinical signs is relatively high. In contrast, detecting infection in asymptomatic animals is problematic. It has been stated that many asymptomatically infected animals may be shedding MAP intermittently in feces ${ }^{2}$ however, the difficulty in culturing dormant organisms may indicate that the problem lies less with intermittent shedding than with intermittent detection. Indeed, evidence of a VNC state in MAP has recently been reported. ${ }^{12}$ The failure to consistently detect infected animals compromises the success of strategies to combat MAP transmission.

Crow and colleagues ${ }^{13}$ described the use of a sterile cytoplasmic membrane preparation obtained from Escherichia coli as a means to reduce oxygen in culture media. This preparation, which is available commercially as Oxyrase $^{\circledR}$ for Broth (OB), has been used successfully to resuscitate VNC entero-hemorrhagic E. coli and Salmonella, ${ }^{14}$ Listeria monocytogenes, ${ }^{15}$ and Campylobacter jejuni. ${ }^{16}$ Reducing oxygen tension in the culture environment may limit oxidative damage and ease the transition from dormancy into active aerobic metabolism during recovery in diagnostic culture procedures. ${ }^{17}$ This could possibly decrease recovery time, allowing for earlier detection of MAP in fecal culture. Earlier detection of MAP infection could thus help to
Correspondence: Timothy E. Secott, Department of Biological Sciences, Minnesota State University, Mankato, 242 Trafton South, Mankato, MN 56001, USA.

Tel.: +1.507.389.2266 - Fax: + 1.507.389.2788.

E-mail: timothy.secott@mnsu.edu

Key words: Mycobacterium; paratuberculosis; dormancy; oxyrase; resuscitation.

Acknowledgements: the authors are grateful for support from Minnesota State University, Mankato (MSUM) Alumni Foundation Grants to KR and LK, an American Society for Microbiology Undergraduate Research Fellowship to KR, and the MSUM Department of Biological Sciences.

Contributions: TS designed experiments, with input from KR and LK. KR and LK performed experiments. KR, LK, and TS performed data analysis and contributed equally to the writing of the manuscript.

Conflict of interest: the authors declare no potential conflict of interest.

Funding: the authors are grateful for support from Minnesota State University, Mankato (MSUM) Alumni Foundation Grants to KR and LK, an American Society for Microbiology Undergraduate Research Fellowship to KR, and the MSUM Department of Biological Sciences.

Received for publication: 27 October 2015 .

Revision received: 10 December 2015.

Accepted for publication: 1 February 2016.

This work is licensed under a Creative Commons Attribution NonCommercial 4.0 License (CC BYNC 4.0).

(C) Copyright K. Rock et al., 2016

Licensee PAGEPress, Italy

Microbiology Research 2016; 7:5695

doi:10.4081/mr.2016.5695

reduce the spread of Johne's disease through and between herds.

\section{Materials and Methods}

\section{Media and bacteria}

Mycobacterium smegmatis $\mathrm{mc}^{2} 155$ (MS) (kindly provided by Richard Groger, Washington University in St. Louis) was cultured in sealed $25 \times 125 \mathrm{~mm}$ tubes containing $17 \mathrm{~mL}$ lysogeny broth (Becton Dickinson, Franklin Lakes, NJ, USA) supplemented with 0.05\% Tween 80 (Becton Dickinson) (LB/Tween) and $1.5 \mathrm{~g} / \mathrm{mL}$ methylene blue (VWR International, Radnor, PA, USA), and incubated with gentle agitation at $37^{\circ} \mathrm{C}$ to 
allow a slow transition to anaerobiosis. .,18 $^{7,18}$ Cultures were used no earlier than 4 days after methylene blue reduction was complete. For starvation dormant (SD) cultures, Mycobacterium paratuberculosis PAMSUM-8, isolated from a dairy cow with clinical paratuberculosis (Minnesota State University, Mankato culture collection) was cultured in sealed $75 \mathrm{~cm}^{2}$ tissue culture flasks containing $30 \mathrm{~mL}$ Middlebrook 7H9 broth (Becton Dickinson) supplemented with 10\% oleic acid, albumin, dextrose, and catalase (Becton Dickinson); $2 \mu \mathrm{g} / \mathrm{mL}$ mycobactin J (Allied Monitor, Fayette, M0, USA); and 0.05\% Tween 80 (M7H9C) and incubated at $37^{\circ} \mathrm{C}$ for at least 9 months before use in experiments. Cultures were used at least 9 months after sealing flasks. To obtain anaerobically dormant (AD) cultures, MAP was cultured in rubber stoppered $30 \mathrm{~mL}$ serum vials containing $20 \mathrm{~mL}$ M7H9C and incubated at $37^{\circ} \mathrm{C}$. In preliminary experiments incorporating $1.5 \mathrm{~g} / \mathrm{mL}$ methylene blue in $\mathrm{M} 7 \mathrm{H} 9 \mathrm{C}$ as a redox indicator, it was observed that complete dye reduction required 8 weeks (Secott and Ghimire 2011, not published). Therefore, AD cultures were held for 3 months before use in experiments.

\section{Preparation of microplates}

Two fold serial dilutions (1:2-1:2048) of OB (Oxyrase, Mansfield, OH, USA) were prepared in the appropriate medium (LB/Tween for MS; M7H9C for MAP). One hundred microliters of each dilution was added to eight wells of a sterile 96-well microplate (Corning, New York, NY, USA) (columns 1-11). The twelfth column contained medium only and therefore served as a negative control. After centrifugation of bacterial cultures at $1600 \times g$ for 20 minutes, cell pellets were resuspended in $0.01 \mathrm{M}$ phosphatebuffered saline (pH 7.4) with $0.05 \%$ Tween 20 (VWR, USA) (PBST). Bacterial suspensions were adjusted to a density equivalent to $2 \times 10^{8}$ colony forming units (CFU)/mL using a colorimeter (Vitek, bioMérieux, Marcy-l'Étoile, France). The cultures were further diluted to a final concentration of $2000 \mathrm{CFU} / \mathrm{mL}$ in the appropriate culture medium, and $100 \mathrm{~L}$ of bacterial suspension was added to microplate wells containing medium only or $\mathrm{OB}$ in medium to yield a final cell density of $1000 \mathrm{cfu} / \mathrm{mL}$. The plate was then covered with a transparent seal (Whatman, Maidstone, UK) and incubated with gentle agitation in a $37^{\circ} \mathrm{C}$ incubator. Optical density (590 nm) was measured using a microplate reader (Thermo Fisher, Waltham, MA, USA) every 24-96 hours until growth ceased or contamination was observed.

\section{Viability estimation}

The BacLight Live/Dead bacterial viability kit (Life Technologies, Thermo Fisher, USA) was used to estimate MAP viability. Included in the kit are SYT0 ${ }^{\circledR} 9$ and propidium iodide (PI) nucleic acid stains. Because intact cytoplasmic membranes are non-permeable to PI, only dead or dying cells will be stained with PI. In contrast, SYTO ${ }^{\circledR} 9$ is membrane-permeable, and cells exhibiting high levels of SYT0 ${ }^{\circledR} 9$ fluorescence were regarded as alive. Attempts were made to prepare a standard curve to measure viability according to the manufacturer's guidelines; however, correlation between expected and observed results was not satisfactory. It was decided to use the ratio of SYTO $^{\circledR} 9$ fluorescence to that of PI to categorize cultures as high $(\geq 0.4)$ or low $(\leq 0.01)$ viability. MAP cultures were harvested by centrifugation as described above and resuspended in PBST to a density equivalent to $1 \times 10^{5}$ CFU/mL. A $0.5 \mathrm{~mL}$ aliquot of the suspension was stained for 10 minutes with an equal volume of a 1:1 mixture of SYT0 ${ }^{\circledR}$ 9:PI prepared according to the manufacturer's instructions. Staining was analyzed using a Guava EasyCyte Mini flow cytometer (Millipore, Merck, Darmstadt, Germany), and cultures were then further diluted for use in $\mathrm{OB}$ resuscitation assays.

\section{Data analysis}

The optical densities of cultures were normalized by averaging the optical density of all wells after experimental setup (typically Day 0 ), and subtracting this value from readings at all subsequent time points. This allowed for the subtraction of the signal given by the medium and initial inoculum alone and therefore quantified only the growth of the organism. Data were analyzed using two-way repeated measures ANOVA and Bonferroni post hoc tests. The number of positive wells within a treatment was also determined, where wells yielding $\mathrm{OD}_{590}$ values greater than 0.085 were defined as positive.

\section{Results}

\section{General comments}

For each of the experiments described below, two-way repeated measures ANOVA revealed significant interactions between time and $\mathrm{OB}$ concentration $(\mathrm{P}<0.0001)$, complicating accurate interpretation of $P$ values corresponding to independent contributions of those variables. The effect of matching was significant $(\mathrm{P}<0.0001)$, supporting the use of the repeated measures ANOVA for analysis.

\section{Oxyrase ${ }^{\circledR}$ for Broth improved the recovery of dormant \\ Mycobacterium smegmatis}

The inclusion of $\mathrm{OB}$ in LB/Tween improved the rate of recovery of $\mathrm{AD}$ MS. However, the concentration of $\mathrm{OB}$ that enhanced resuscita- tion varied between experiments. In the first experiment with MS, significant $(\mathrm{P}<0.05$ for Bonferroni post hoc tests) improvement in recovery was observed on days 2-4 in cultures treated with 1:2 $0 \mathrm{~B}$, day 3 in those treated with $1: 4 \mathrm{OB}$, and days 3 and 4 for those treated with 1:8 OB (Figure 1A). OB also increased the number of wells that were growth-positive and reduced the time needed for recovery for MS treated with 1:2, 1:4, and 1:8 OB (Figure 2A).

In the second experiment, low dilutions (high concentrations) had no effect on the recovery of dormant MS (data not shown). Instead, enhanced resuscitation was observed only when intermediate concentrations of $\mathrm{OB}$ were used (Figure 1B). There was a drop in the mean optical density for all cultures on day 6 . Statistically significant enhancement of dormant MS recovery was seen on day 12 for cultures treated with $1: 128$ OB $(\mathrm{P}<0.05$ for Bonferroni post hoc tests). Again, OB increased the number of culture-positive wells and reduced the time needed to reach positive status for MS cultures treated with 1:64 and 1:128 0B (Figure 2B).

\section{Oxyrase ${ }^{\circledR}$ for Broth improved the recovery of dormant Mycobacterium avium subsp. paratuberculosis}

M7H9C supplemented with OB improved the rate of recovery of SD MAP (Figure 3), but as was the case for MS, the concentration of $\mathrm{OB}$ that effected maximal recovery differed between experiments. Intermediate-to-low concentrations of $\mathrm{OB}$ were observed to improve the resuscitation of MAP in the first experiment, with significant differences between the control and 1:512 OB-treated cultures on days 4 and $5(\mathrm{P}<0.05$ for Bonferroni post hoc tests) (Figure 3A). Time to detection was reduced and an accompanying increase in the number of positive wells was seen in cultures treated with 1:256 and 1:512 OB (Figure 4A). OB significantly enhanced the rate of recovery of SD MAP in cultures treated with $1: 2,1: 4$, and 1:8 OB in the second experiment for days 2-20 $(\mathrm{P}<0.01$ for Bonferroni post hoc tests) (Figure $3 \mathrm{~B}$ ). Medium containing these dilutions of $\mathrm{OB}$ also increased the number of SD MAP cultures that were resuscitated and reduced the time needed to reach positivity (Figure 4B).

$\mathrm{OB}$ also enhanced the resuscitation of $\mathrm{AD}$ MAP cultures (Figure 5). These observations are discussed in detail below.

\section{Seed culture viability influences the concentration of Oxyrase ${ }^{\circledR}$ for Broth needed for improved resuscitation}

While OB improved the recovery of dormant 
MS and MAP, the concentration of $\mathrm{OB}$ required for maximal resuscitation varied from experiment to experiment. Because the concentrations of MS and MAP were estimated by turbidimetry, which does not distinguish between dead and live organisms, it was suspected that differences in OB-promoted resuscitation reflected differences in seed culture viability. To further investigate this, seed culture viability was estimated in parallel with the setup of experiments using AD MAP.

High viability AD MAP cultures responded best to high concentrations (low dilutions) of OB (Figure 5A). In this experiment, medium containing 1:2 OB exhibited improved resuscitation on days 12 and 14 as compared to the control, although this effect was not significant ( $\mathrm{P}>0.05$ for Bonferroni post hoc tests). Similar to what was described above for Figure $1 \mathrm{~B}$, the mean optical density dropped to or remained at 0.0 on day 7 for all cultures. In contrast, low viability cultures yielded enhanced recovery only in medium containing low concentrations of $\mathrm{OB}$ (Figure 5B). Here,
Bonferroni post hoc tests indicated a significant $(\mathrm{P}<0.01)$ improvement in recovery for $\mathrm{AD}$ MAP grown in the presence of 1:2048 OB on day 11 as compared to the control.

\section{Discussion}

Fecal culture is regarded as the gold standard for ante mortem diagnosis of MAP infection. However, the overall sensitivity of MAP diagnostic culture is $33 \%{ }^{3}$ This is a consequence of the inability to consistently detect infection in asymptomatically infected cattle. This limitation must be overcome if the chances for success of Johne's disease control programs are to be improved. To this end, it was the purpose of this study to evaluate the potential for the inclusion of $\mathrm{OB}$ in culture media to improve MAP diagnostic culture sensitivity. Because MS has been previously used in dormancy studies, ${ }^{18-20}$ it was used in this investigation for proof of principle. Indeed, $\mathrm{OB}$ increased the rate of recovery and growth yield for dormant MS and dormant MAP. To our knowledge, this is the first report in peerreviewed literature to document the ability of $\mathrm{OB}$ to improve the resuscitation of dormant mycobacteria.

Resuscitation-promoting factors (Rpf), which were originally identified in Micrococcus luteus, ${ }^{21}$ have been shown to improve the recovery of dormant mycobacteria in several investigations. ${ }^{22-24}$ Several open reading frames corresponding to Rpf homologues observed in M. tuberculosis have been identified in the genome sequence of MAP. ${ }^{25}$ One of the events seen in the transition of mycobacteria to dormancy is the thickening of the cell wall. ${ }^{26}$ Lamont and colleagues described the presence of spore-like morphotypes in dormant cultures of MAP, which would be consistent with the appearance of cell wall thickening. ${ }^{27}$ As Rpf proteins are believed to have the ability to hydrolyze peptidoglycan, ${ }^{28}$ it is tempting to speculate that these proteins also play a role in the resuscitation of MAP.
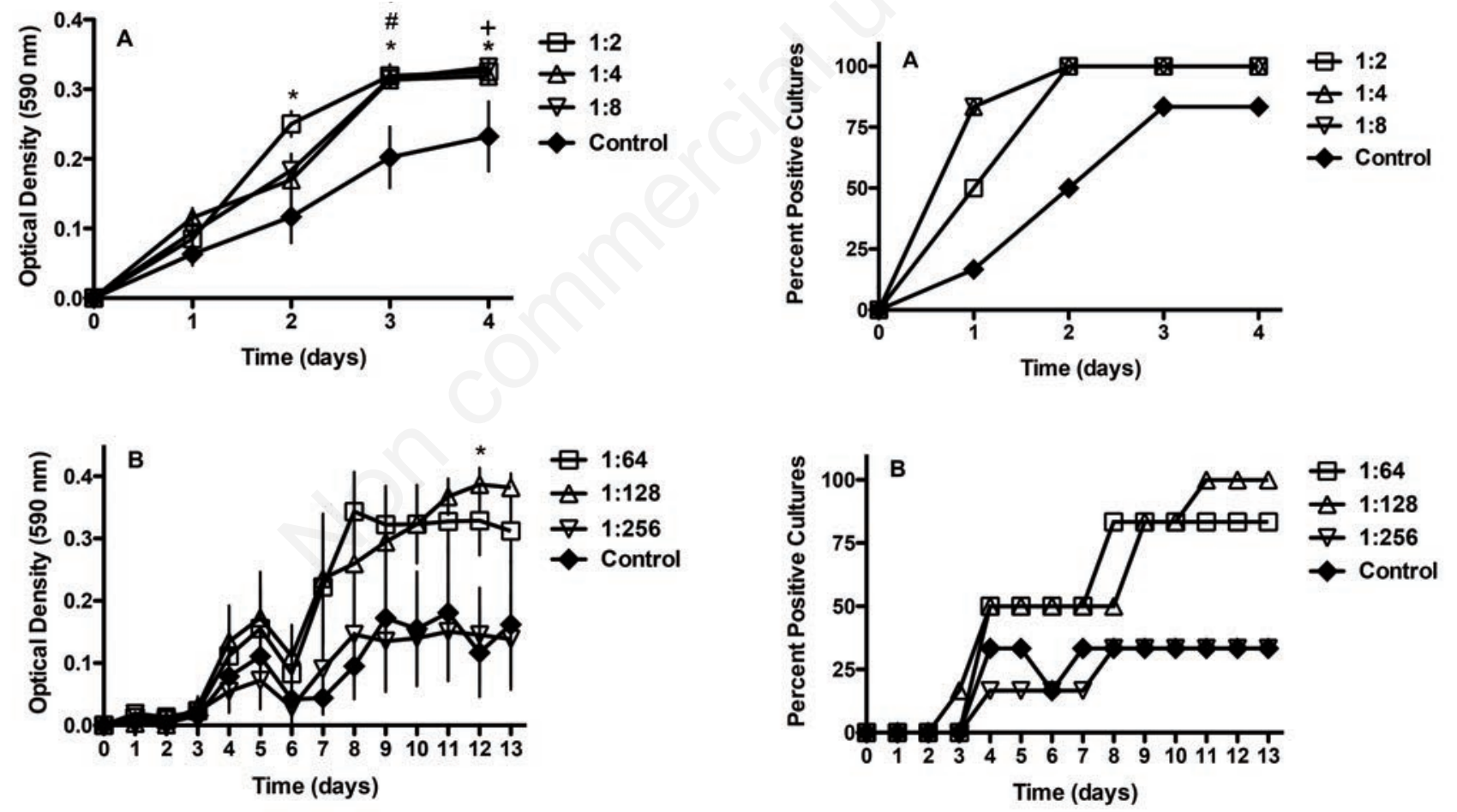

Figure 1. Inclusion of $\mathrm{Oxyrase}^{\circledast}$ for Broth $(\mathrm{OB})$ in culture medium enhances the rate of recovery of Mycobacterium smegmatis (MS). The optical density (590 $\mathrm{nm}$ ) of MS grown in LB broth containing serial dilutions of $\mathrm{OB}$ was measured until growth ceased or contamination was observed. The average optical density all inoculated wells on day 0 was subtracted from that of each at all subsequent time points. Only the dilutions of $O B$ that affected MS growth are shown. A) Experiment 1. Asterisks, pound signs, and pluses indicate significant differences between controls and 1:2, 1:4, and 1:8 OB-treated cultures, respectively $(\mathrm{P}<0.05)$. B) Experiment 2. Asterisks indicate significant differences between controls and 1:128 OB-treated cultures $(P<0.05)$.

Figure 2. Oxyrase for Broth (OB) increases the number of Mycobacterium smegmatis (MS) cultures that recover from dormancy. The optical density (590 $\mathrm{nm}$ ) of MS grown in LB broth containing serial dilutions of $\mathrm{OB}$ was measured until growth ceased or contamination was observed. Wells were regarded positive if the optical density was greater than $0.085 \mathrm{OD}_{590}$ units greater than the plate average at day 0 . Only the dilutions of $\mathrm{OB}$ that affected MS growth are shown. A) Experiment 1. B) Experiment 2. 
Nevertheless, the observation that $\mathrm{OB}$ promoted recovery of dormant MAP indicates that the degree of oxidative damage that occurs when dormant mycobacteria resume growth in rich media, such as those used in diagnostic culture, is an important consideration that may influence the outcome of diagnostic culture, particularly in cases where low numbers of organisms are present in the specimen.

While the recovery of dormant mycobacteria was clearly enhanced by the inclusion of $\mathrm{OB}$ in culture media, the concentration that led to this enhancement varied considerably among all trials, and this was likely due to differences in the concentration of viable organisms in dormant cultures. Others have observed considerable differences in dormant MAP culture viability. ${ }^{29}$ Votyakova and colleagues ${ }^{30}$ observed that in order for resuscitation of dormant Micrococcus luteus to occur, that population of cells had to contain a subpopulation of at least 9 actively growing cells, suggesting that these actively growing cells were a source of some factor that acted in a paracrine manner to effect resuscitation. It is possible that high concentrations of $\mathrm{OB}$ prevent the oxidative metabolism of the very small number of actively growing MAP present in low viability dormant cultures, and that these resuscitative paracrine factors can be produced in sufficient quantity only under low $\mathrm{OB}$ concentrations.

Organisms such as Listeria monocytogenes, Salmonella enterica, and Escherichia coli, which have been successfully resuscitated from the VNC state through the use of $\mathrm{OB},{ }^{14,15}$ are facultative anaerobes. In the presence of $\mathrm{OB}$, these organisms likely ferment or respire anaerobically. Like Micrococcus, mycobacteria are considered obligate aerobes; yet, it has been demonstrated that $\mathrm{AD}$ mycobacteria survive using a form of nitrate reductase, ${ }^{20,31}$ which fuels a gene expression profile distinct from that of actively growing mycobacteria. ${ }^{32}$ Nevertheless, a functional nitrate reductase is not present in the genome of MAP. ${ }^{25}$ How these organisms are able to metabolize in the pres- ence of $\mathrm{OB}$, particularly at high concentrations, has yet to be elucidated. The sudden drop in optical density that was reported herein for two experiments is unlikely to have represented a corresponding death of MAP. Optical density does not distinguish between live and dead cells, and MAP is unlikely to have lysed during that short window of time. Rather, we suspect that the drop in optical density represents the consequence of a technical error, such as reading the microplate with condensation on the plastic film covering the plate, which was magnified during the normalization process.

Because the concentration of $\mathrm{OB}$ needed to yield efficient resuscitation of MAP requires foreknowledge of the number of viable organisms present in the sample, it is not anticipated that the application of $\mathrm{OB}$ as a medium supplement for routine diagnostic culture will occur in the near future. Still, this approach may prove useful diagnostic testing of animals prior to sale or breeding.
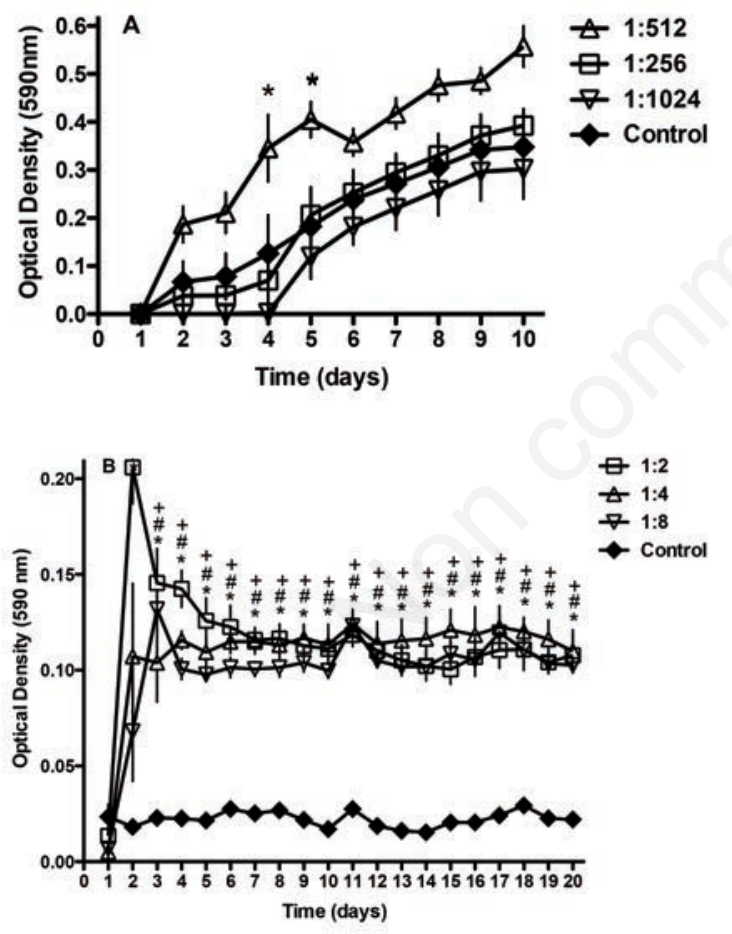

Figure 3. Oxyrase ${ }^{\circledast}$ for Broth (OB) enhances the recovery of Mycobacterium avium subsp. paratuberculosis (MAP). The optical density $(590 \mathrm{~nm})$ of MAP grown in Middlebrook 7H9C containing serial dilutions of $O B$ was measured until growth ceased or contamination was observed. The average optical density of all inoculated wells on day 0 was subtracted from that of each well at all subsequent time points. Only the dilutions of $O B$ that affected MAP growth are shown. A) Experiment 1. Asterisks indicate significant differences between controls and 1:512 OB-treated cultures $(P<0.05)$. B) Experiment 2. Asterisks, pound signs, and pluses indicate significant differences between controls and 1:2, 1:4, and 1:8 OB-treated cultures, respectively $(\mathrm{P}<0.0001)$.
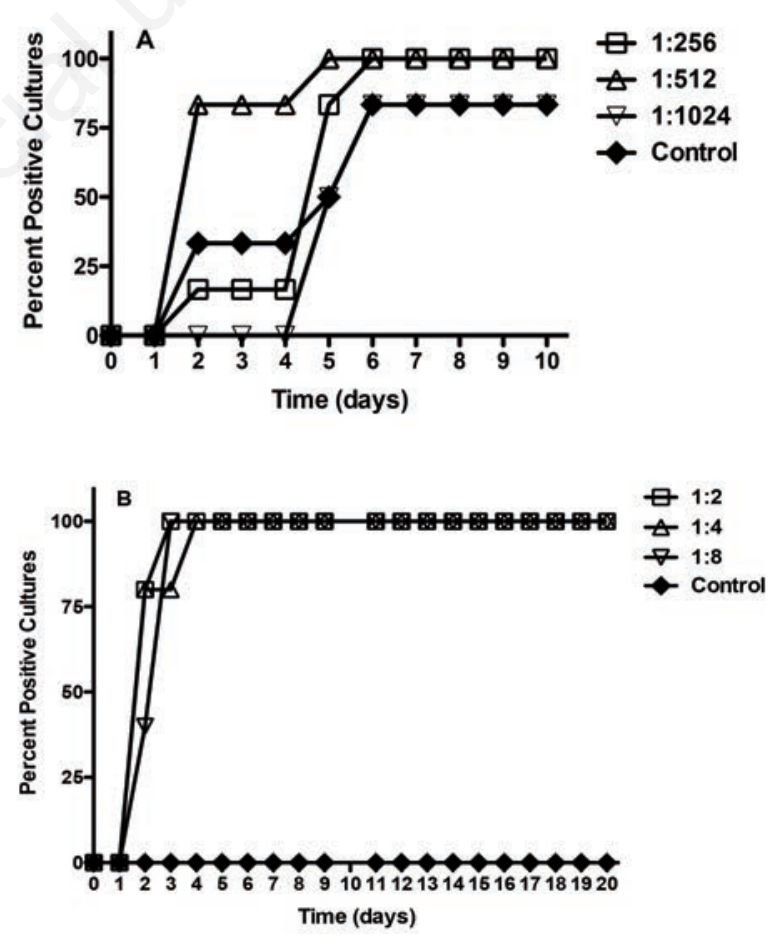

Figure 4. Oxyrase ${ }^{\circledast}$ for Broth (OB) increases the number of Mycobacterium avium subsp. paratuberculosis (MAP) cultures that recover from dormancy. The optical density $(590 \mathrm{~nm})$ of MAP grown in Middlebrook 7H9C containing serial dilutions of $O B$ was measured daily until growth ceased or contamination was observed. Wells were regarded positive if the optical density was greater than $0.085 \mathrm{OD}_{590}$ units greater than the plate average at day 0 . Only the dilutions of $O B$ that affected MAP growth are shown. A) Experiment 1. B) Experiment 2. 

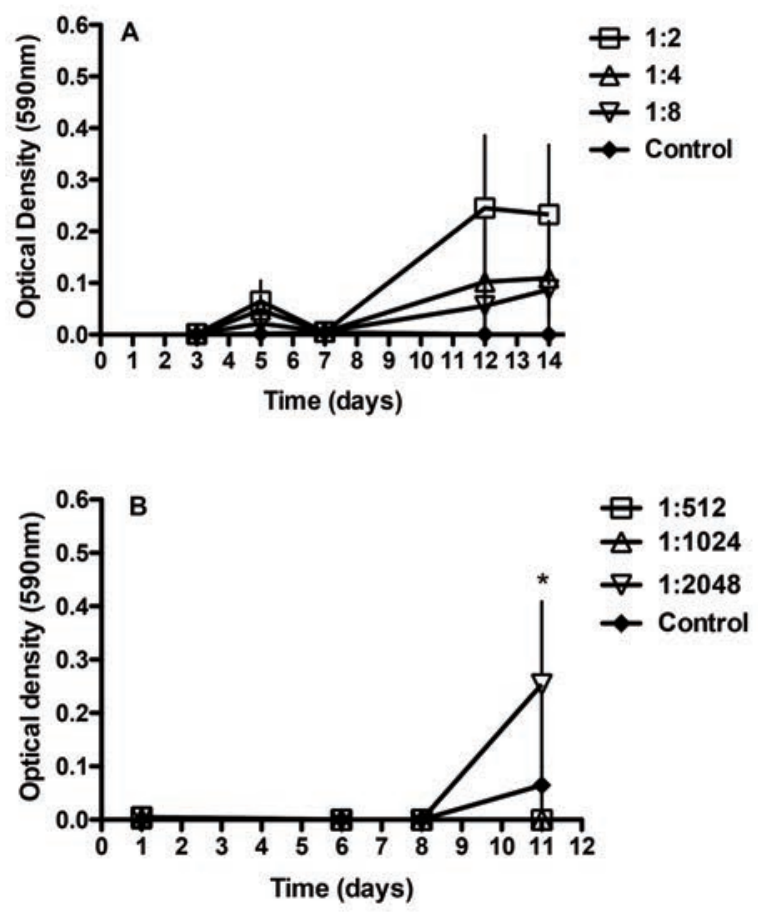

Figure 5. The concentration of Oxyrase ${ }^{\circledast}$ for Broth (OB) that enhances the recovery of dormant Mycobacterium avium subsp. paratuberculosis (MAP) is influenced by the number of viable organisms present. The optical density $(590 \mathrm{~nm}$ ) of MAP grown in Middlebrook $7 \mathrm{H} 9 \mathrm{C}$ containing serial dilutions of $\mathrm{OB}$ was measured every 2-4 days until growth ceased or contamination was observed. Only the dilutions of $O B$ that affected MAP growth are shown. A) Growth of organisms in plates prepared from a high viability dormant culture. B) Growth of organisms in plates prepared from a low viability dormant culture. The asterisk indicates a significant difference between the control cultures and those treated with 1:2048 OB $(\mathrm{P}<0.05)$.

\section{Conclusions}

Efforts to control the spread of Johne's disease are compromised by the poor sensitivity of diagnostic fecal culture for MAP. Modification of fecal culture media and protocols to enable the consistent recovery of dormant MAP must be developed in order to improve fecal culture sensitivity. While $\mathrm{OB}$ can potentially be used to resuscitate dormant MAP, the inclusion of this reagent in culture protocols is presently hampered by the observation that the number of viable MAP in feces determines the concentration of $\mathrm{OB}$ needed to promote the recovery of dormant MAP in culture. Further, it remains to be established whether the resuscitative effect reported herein for OB also applies to other MAP strains. The MAP PAMSUM-8 strain has phenotypic characteristics most consistent with those of MAP cattle (C, or type II) strains (Secott 2003, not published). Whether this strain is representative of the response of $\mathrm{C}$ strains, sheep (S, or type I and type III) strains, or all strains will require further testing. ${ }^{33} \mathrm{OB}$ may prove useful for improving culture sensitivity once mycobacterial dormancy is more thoroughly characterized. However, the use of $\mathrm{OB}$ to promote the resuscitation of MAP should be evaluated by testing fecal samples from animals with known MAP infection status, employing all of the culture media currently used for diagnostic culture. Whether $\mathrm{OB}$ acts directly on recovering cells to promote resuscitation by preventing oxidative damage, or acts in concert with other factors has yet to be determined.

\section{References}

1. Ott SL, Wells SJ, Wagner BA. Herd-level economic losses associated with Johne's disease on US dairy operations. Prev Vet Med 1999;40:179-92.

2. Whitlock RH, Buergelt C. Preclinical and clinical manifestations of paratuberculosis (including pathology). Vet Clin North Am Food Anim Pract 1996;12:345-56.

3. Whitlock RH, Wells SJ, Sweeney RW, Van Tiem J. ELISA and fecal culture for paratuberculosis (Johne's disease): sensitivity and specificity of each method. Vet Microbiol 2000;77:387-98.

4. Magombedze G, Ngonghala CN, Lanzas C. Evalution of the "iceberg phenomenon" in Johne's disease through mathematical modelling. PLoS ONE 2013;8:e76636.

5. Sweeney RW. Transmission of paratuberculosis. Vet Clin North Am Food Anim Pract 1996;12:305-12.

6. Sweeney RW. Pathogenesis of paratuberculosis. Vet Clin North Am Food Anim Pract 2011;27:537-46.

7. Wayne LG, Hayes LG. An in vitro model for sequential study of shiftdown of Mycobacterium tuberculosis through two stages of nonreplicating persistence. Infect Immun 1996;64:2062-9.

8. Whittington RJ, Marshall DJ, Nicholls PJ, et al. Survival and dormancy of Mycobacterium avium subsp. paratuberculosis in the environment. Appl Environ Microbiol 2004;70:2989-3004.

9. Whittington RJ, Marsh IB, Reddacliff LA. Survival of Mycobacterium avium subsp. paratuberculosis in dam water and sediment. Appl Environ Microbiol 2005;71: 5304-8.

10. Oliver JD. The viable but nonculturable state in bacteria. J Microbiol 2005;43:93100.

11. Wang H-W, Chung C-H, Ma T-Y, Wong H-C. Roles of alkyl hydroperoxide reductase subunit C (AhpC) in viable but nonculturable Vibrio parahaemolyticus. Appl Environ Microbiol 2013;79:3734-43.

12. Nazareth N, Magro F, Appelberg R, et al. Increased viability but decreased culturability of Mycobacterium avium subsp. paratuberculosis in macrophages from inflammatory bowel disease patients under infliximab treatment. Med Microbiol Immunol 2015;204:647-56.

13. Crow WD, Machanoff R, Adler HI. Isolation of anaerobes using an oxygen reducing membrane fraction: experiments with acetone butanol producing organisms. J Microbiol Methods 1985;4:133-9.

14. Reissbrodt R, Rienaecker I, Romanova JM, et al. Resuscitation of Salmonella enterica serovar typhimurium and enterohemorrhagic Escherichia coli from the viable but nonculturable state by heat-stable enterobacterial autoinducer. Appl Environ Microbiol 2002;68:4788-94.

15. Patel JR, Beuchat LR. Enrichment in Fraser broth supplemented with catalase or Oxyrase, combined with the microcolony immunoblot technique, for detecting heat-injured Listeria monocytogenes in foods. International J Food Microbiol 1995;26:165-76.

16. Tran TT. Evaluation of Oxyrase enrichment method for isolation of Campylobacter jejuni from inoculated 
foods. Lett Appl Microbiol 1995;21:345-7.

17. Reissbrodt R, Rassbach A, Burghardt B, et al. Assessment of a new selective chromogenic Bacillus cereus group plating medium and use of enterobacterial autoinducer of growth for cultural identification of Bacillus species. J Clin Microbiol 2004;42:3795-8.

18. Dick T, Lee BH, Murugasu-0ei B. Oxygen depletion induced dormancy in Mycobacterium smegmatis. FEMS Microbiol Lett 1998;163:159-64.

19. Hutter B, Dick T. Increased alanine dehydrogenase activity during dormancy in Mycobacterium smegmatis. FEMS Microbiol Lett 1998;167:7-11.

20. Hutter B, Dick T. Up-regulation of narX, encoding a putative "fused nitrate reductase" in anaerobic dormant Mycobacterium bovis BCG. FEMS Microbiol Lett 1999;178:63-9.

21. Mukamolova GV, Kaprelyants AS, Young DI, et al. A bacterial cytokine. Proc Natl Acad Sci USA 1998;95:8916-21.

22. Mukamolova GV, Turapov OA, Young DI, et al. A family of autocrine growth factors in Mycobacterium tuberculosis. Mol Microbiol 2002;46:623-35.

23. Shleeva MO, Mukamolova GV, Telkov MV, et al. Formation of nonculturable cells of
Mycobacterium tuberculosis and their resuscitation. Microbiology 2003;72:64-70.

24. Shleeva M, Mukamolova GV, Young M, et al. Formation of "non-culturable" cells of Mycobacterium smegmatis in stationary phase in response to growth under suboptimal conditions and their Rpf-mediated resuscitation. Microbiology 2004;150: 1687-97.

25. Li L, Bannantine JP, Zhang Q, et al. The complete genome sequence of Mycobacterium avium subspecies paratuberculosis. Proc Natl Acad Sci USA 2005;102:12344-9.

26. Cunningham AF, Spreadbury CL. Mycobacterial stationary phase induced by low oxygen tension: cell wall thickening and localization of the 16-kilodalton alphacrystallin homolog. J Bacteriol 1998;180: 801-8.

27. Lamont EA, Bannantine JP, Armién A, et al. Identification and characterization of a spore-like morphotype in chronically starved Mycobacterium avium subsp. paratuberculosis cultures. PLoS ONE 2012;7:e30648.

28. Mukamolova GV, Murzin AG, Salina EG, et al. Muralytic activity of Micrococcus luteus Rpf and its relationship to physiological activity in promoting bacterial growth and resuscitation. Mol Microbiol 2006;59:8498.

29. Gumber S, Whittington RJ. Analysis of the growth pattern, survival and proteome of Mycobacterium avium subsp. paratuberculosis following exposure to heat. Vet Microbiol 2009;136:82-90.

30. Votyakova TV, Kaprelyants AS, Kell DB. Influence of viable cells on the resuscitation of dormant cells in Micrococcus luteus cultures held in an extended stationary phase: the population effect. Appl Environ Microbiol. 1994;60:3284-91.

31. Sohaskey CD. Regulation of nitrate reductase activity in Mycobacterium tuberculosis by oxygen and nitric oxide. Microbiology 2005;151:3803-010.

32. Devasundaram S, Khan I, Kumar N, et al. The influence of reduced oxygen availability on gene expression in laboratory (H37Rv) and clinical strains (S7 and S10) of Mycobacterium tuberculosis. J Biotech 2015;210:70-80.

33. Möbius $P$, Hölzer $M$, Felder $M$, et al. Comprehensive insights in the Mycobacterium avium subsp. paratuberculosis genome using new WGS data of sheep strain JIII-386 from Germany. Genome Biol Evol 2015;7: 2585-601. 